ESAIM: PROCEEDINGS, September 2005, Vol.14, 63-71

Eric Cancès \& Jean-Frédéric Gerbeau, Editors

DOI: $10.1051 /$ proc:2005006

\title{
BOUNDARY ELEMENT FORMULATION FOR ELECTRICAL IMPEDANCE TOMOGRAPHY
}

\author{
M. Clerr ${ }^{1}$, J.-M. Badier ${ }^{2}$, G. Adde ${ }^{1}$, J. KybiC ${ }^{3}$ And T. Papadopoulo ${ }^{1}$
}

\begin{abstract}
This paper concerns the modelisation and simulation of the forward problem of Electrical Impedance Tomography (EIT), i.e. the computation of an electrical potential due to an applied boundary current. The underlying goal is the estimation of the conductivities of the head tissues, in particular the skull, in order to improve the head models used in Electroencephalography (EEG). In the quasistatic approximation, the problem can be modeled by a Poisson equation with a non-vanishing Neumann boundary condition. We introduce a symmetric boundary integral formulation, which is discretized using mixed finite elements, and show its application to an EIT experiment in view of estimating skull conductivity.
\end{abstract}

Résumé. Nous nous intéressons à la modélisation et la simulation du problème direct de la Tomographie par Impédance Électrique (EIT), i.e. le calcul du potentiel électrique dans un volume résultant de l'application d'un courant sur la surface. Le but sous-jacent est l'estimation des conductivités des tissus de la tête, en particulier celle du crâne, afin d'améliorer les modèles de tête utilisés en Electroencéphalographie (EEG). Par une approximation quasistatique, on modélise le problème par une équation de Poisson, avec condition de Neumann non-nulle sur le bord. Nous introduisons une formulation symétrique par équations intégrales, ainsi que sa discrétisation par éléments finis mixtes. Nous montrons l'application de cette méthode à une expérience d'EIT, en vue d'estimer la conductivité du crâne.

\section{INTRODUCTION}

Electroencephalography (EEG) concerns the passive measurement of electrical potential on a subject's scalp. It can be used to estimate the spatial distribution of electrical activity in the brain, a source estimation problem from boundary measurements, known as the inverse EEG problem. The forward EEG problem, modelled by a Poisson equation, relates a supposedly known current distribution to the potential.

Originally, solutions to the forward EEG problem were proposed for a simplified head model made of concentric spheres of different conductivities, representing the different tissues composing the head. With such models, analytical solutions based on spherical harmonic expansions allow to compute the potential, for a current source modelled as the superposition of isolated dipoles.

It has become possible to develop subject dependent geometrical models, in which the interfaces between tissues of different conductivities are obtained from the segmentation of the subject's Magnetic Resonance

\footnotetext{
1 Odyssée Laboratory, ENPC-ENS Ulm-INRIA, France

e-mail: Maureen.Clerc@sophia.inria.fr, Theo.Papadopoulo@sophia.inria.fr, adde@certis.enpc.fr

${ }^{2}$ Laboratoire de Neurophysiologie et Neuropsychologie, INSERM Université de la Méditerranée, Marseille, France

${ }^{3}$ Center for Machine Perception, CTU, Prague, Czech Republic

e-mail: kybic@fel.cvut.cz
}

(c) EDP Sciences, SMAI 2005 
Image (MRI). The forward EEG problem is solved using either Finite Difference, Boundary Element or Finite Element Methods (ordered in increasing complexity in terms of geometrical description). The Boundary Element Method assumes that the conductivity is piecewise constant, and uses discretizations based on the meshes of the interfaces between tissues. The Finite Difference or the Finite Element Methods, which are based on volumic meshes, can assume conductivity distributions with more intricate variations, including anisotropic, tensor-valued conductivity fields.

A major difficulty with any of these methods is to decide which conductivity value to assign to each tissue. This is all the more crucial as the inverse source problem is sensitive to the tissue conductivity values [9].

Electrical Impedance Tomography (EIT) has recently been shown appropriate for estimating the values of tissue conductivities inside the head, particularly the skull-to-scalp conductivity ratio $[1,5,7]$. Its principle is to impose a (very small) source of current on the scalp, and to measure the resulting electrical potential on the scalp by EEG. The conductivity values are estimated by minimizing the difference between the measurements and the potential predicted by the forward EIT problem.

The purpose of this article is to present an original solution to the forward EIT problem, based on a symmetric Boundary Element Method, whose high accuracy for the forward electroencephalography (EEG) problem has recently been reported [10]. The article is organised as follows. Section 2 presents the EEG and EIT models: Poisson equations which only differ by the boundary condition on the scalp. Section 3 recalls the fundamental Representation Theorem which is the backbone of the symmetric Boundary Element method. Section 4 establishes the symmetric boundary integral formulation, and its discretization is detailed in Section 5, along with the validation procedure used. Finally, results of simulations on a realistic head model are presented in Section 6, indicating its appropriateness for solving the inverse EIT problem.

\section{EEG AND EIT MODELS}

At the frequencies of interest for EEG (below $1 \mathrm{kHz}$ ), the quasistatic regime applies for the electromagnetic propagation inside the head volume [8]. The current density is therefore divergence-free $\nabla \cdot J=0$. A macroscopic model for current density in the brain is the combination of an ohmic contribution and a primary source contribution (the current due to the post-synaptic potential discharges)

$$
J=\sigma E+\mathbf{J}^{\mathrm{p}}
$$

In the quasistatic approximation, the electric field $E$ derives from a potential $E=-\nabla V$. This electric potential satisfies, inside the head volume $\Omega$, a Poisson equation [14]

$$
\nabla \cdot(\sigma \nabla V)=f=\nabla \cdot \mathbf{J}^{\mathrm{p}} \text { in } \Omega
$$

with the following boundary condition on the scalp:

$$
\sigma \partial_{\mathbf{n}} V=j \text { on } \partial \Omega
$$

In an EEG model, the current on the scalp, $j$, vanishes because of the non-conducting medium (air) around the head. Conversely, in our EIT model, sources inside the head are assumed negligible $(f=0)$ and a non-vanishing current $j$ on the scalp is imposed.

To fix ideas, this paper considers a three-layer geometrical model for the head. Conductivities of each domain are respectively denoted $\sigma_{1}, \sigma_{2}$ and $\sigma_{3}$. The surfaces enclosing these homogeneous conductivity regions are denoted $S_{1}$ (inner skull boundary), $S_{2}$ (skull-scalp interface) and $S_{3}$ (scalp-air interface). 


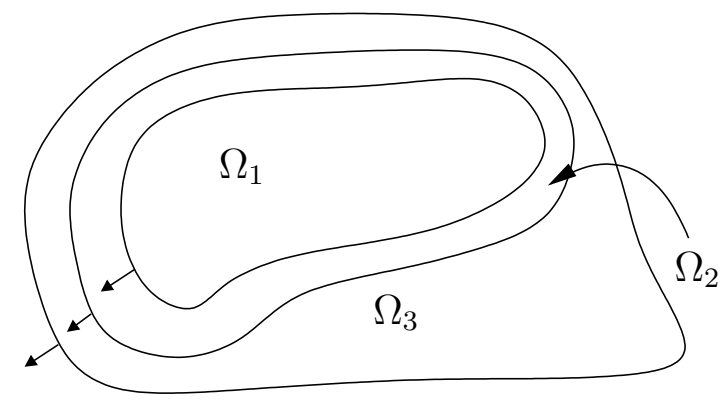

Figure 1. The head is modelled as a set of three nested regions with constant conductivities $\sigma_{1}, \sigma_{2}, \sigma_{3}$. The brain and cerebro-spinal fluid are represented by $\Omega_{1}$, the skull by $\Omega_{2}$ and the scalp by $\Omega_{3}$. The three volumes are delimited by surfaces $S_{1}, S_{2}, S_{3}$, and the normal direction to each surface is indicated by outward-pointing arrows.

Since the conductivity is supposed to be piecewise constant, we can factor out $\sigma$ from (1) to yield a set of Poisson problems coupled by boundary conditions

$$
\begin{aligned}
\sigma_{i} \Delta V=f & & \text { in } \Omega_{i}, \text { for } i=1,2,3 \\
{[V]_{j}=\left[\sigma \partial_{\mathbf{n}} V\right]_{i}=0 } & & \text { on } S_{i}, i=1,2 \\
\sigma \partial_{\mathbf{n}} V=j & & \text { on } S_{3} .
\end{aligned}
$$

\section{Representation Theorem}

The power of the Boundary Element Method is in its conciseness, since it only uses unknowns defined on surfaces rather than in the volume. The key to this dimension reduction resides in the fundamental Representation xTheorem $[2,11]$, which we recall in this section. We first introduce some notation and definitions. The Green function in $\mathbb{R}^{3}$,

$$
G(\mathbf{r})=\frac{1}{4 \pi\|\mathbf{r}\|}
$$

satisfies $-\Delta G=\delta_{0}$, where $\delta_{0}$ is the Dirac distribution at $\mathbf{r}=0$. Given a regular oriented boundary (surface) $\partial \Omega$, we denote by $\mathbf{n}$, resp. $\mathbf{n}^{\prime}$ the outward normal vector at position $\mathbf{r}$, resp. $\mathbf{r}^{\prime}$. The four classical integral operators $\mathcal{D}$ (double-layer), $\mathcal{S}$ (single-layer), $\mathcal{N}$, and $\mathcal{D}^{*}$ (adjoint of $\mathcal{D}$ with respect to the $L^{2}(\partial \Omega)$ scalar product) map a scalar function $f$ on $\partial \Omega$ to another scalar function on $\partial \Omega$ :

$$
\begin{array}{ll}
(\mathcal{D} f)(\mathbf{r})=\int_{\partial \Omega} \partial_{\mathbf{n}^{\prime}} G\left(\mathbf{r}-\mathbf{r}^{\prime}\right) f\left(\mathbf{r}^{\prime}\right) \mathrm{d} s\left(\mathbf{r}^{\prime}\right), & (\mathcal{S} f)(\mathbf{r})=\int_{\partial \Omega} G\left(\mathbf{r}-\mathbf{r}^{\prime}\right) f\left(\mathbf{r}^{\prime}\right) \mathrm{d} s\left(\mathbf{r}^{\prime}\right) \\
(\mathcal{N} f)(\mathbf{r})=\int_{\partial \Omega} \partial_{\mathbf{n}, \mathbf{n}^{\prime}} G\left(\mathbf{r}-\mathbf{r}^{\prime}\right) f\left(\mathbf{r}^{\prime}\right) \mathrm{d} s\left(\mathbf{r}^{\prime}\right), & \left(\mathcal{D}^{*} f\right)(\mathbf{r})=\int_{\partial \Omega} \partial_{\mathbf{n}} G\left(\mathbf{r}-\mathbf{r}^{\prime}\right) f\left(\mathbf{r}^{\prime}\right) \mathrm{d} s\left(\mathbf{r}^{\prime}\right) .
\end{array}
$$

To simplify the treatment and avoid ambiguity, we work with potentials which vanish at infinity; we say that a function $u$ satisfies condition $\mathscr{H}$, if simultaneously

$$
\left\{\begin{array}{l}
\lim _{r \rightarrow \infty} r|u(\mathbf{r})|<\infty \\
\lim _{r \rightarrow \infty} r \frac{\partial u}{\partial r}(\mathbf{r})=0
\end{array}\right.
$$


where $r=\|\mathbf{r}\|$, and $\frac{\partial u}{\partial r}(\mathbf{r})$ denotes the partial derivative of $u$ in the radial direction. The Green function $G$ in (6) satisfies $\mathscr{H}$.

Lastly, we define the jump of a function $f: \mathbb{R}^{3} \rightarrow \mathbb{R}$ across a surface $S$ as

$$
[f]_{S}=f_{S}^{-}-f_{S}^{+},
$$

where the functions $f_{S}^{-}$and $f_{S}^{+}$on $S$ denote respectively the interior and exterior limits of $f$ with respect to the normal orientation

$$
\text { for } \mathbf{r} \in S, \quad f_{S}^{ \pm}(\mathbf{r})=\lim _{\alpha \rightarrow 0^{ \pm}} f(\mathbf{r}+\alpha \mathbf{n}) .
$$

We are now ready to state the fundamental representation theorem:

Theorem 3.1 (Representation Theorem). Let $\Omega \subseteq \mathbb{R}^{3}$ be a bounded open set with a regular boundary $\partial \Omega$. Let $u:\left(\mathbb{R}^{3} \backslash \partial \Omega\right) \rightarrow \mathbb{R}$ be a harmonic function $\left(\Delta u=0\right.$ in $\left.\mathbb{R}^{3} \backslash \partial \Omega\right)$, satisfying the $\mathscr{H}$ condition, and let further $p(\mathbf{r}) \stackrel{\text { def }}{=} \partial_{\mathbf{n}} u(\mathbf{r})$. Then for $\mathbf{r} \in \partial \Omega$

$$
\begin{aligned}
& -\partial_{\mathbf{n}} u^{ \pm}=\quad \mathcal{N}[u]_{\partial \Omega}+\left( \pm \frac{\mathcal{J}}{2}-\mathcal{D}^{*}\right)\left[\partial_{\mathbf{n}} u\right]_{\partial \Omega} \\
& u^{ \pm}=\left(\mp \frac{\mathcal{J}}{2}-\mathcal{D}\right)[u]_{\partial \Omega} \quad+\mathcal{S}\left[\partial_{\mathbf{n}} u\right]_{\partial \Omega}
\end{aligned}
$$

where $\mathcal{J}$ denotes the identity operator.

Theorem 3.1 holds in particular for a hollow ball topology, in which $\partial \Omega$ is composed of two disjoint components. This result will be used in the next section to establish the symmetric formulation.

\section{Symmetric Boundary integral FORMUlation}

We build upon a formulation which has recently been proposed for EEG in [10], extending it to deal with the boundary conditions of EIT. The symmetric integral formulation, which we establish in this section, is based on the work of Nédélec [11]. In this approach, we consider for $i=1,2,3$ a function $u_{i}$ which is harmonic in $\mathbb{R}^{3} \backslash \partial \Omega_{i}$ :

$$
u_{i}=\left\{\begin{array}{ll}
V & \text { in } \Omega_{i} \\
0 & \text { in } \mathbb{R}^{3} \backslash \bar{\Omega}_{i}
\end{array} .\right.
$$

Considering the nested volume model (Fig. 1), the boundary of $\Omega_{i}$ is $\partial \Omega_{i}=S_{i-1} \cup S_{i}$ (with $S_{0}=\emptyset$ ). With respect to the orientations of normals indicated in Fig. 2, the jumps of $u_{i}$ across $S_{i}$ satisfy the relations

$$
\left[u_{i}\right]_{S_{i}}=V_{S_{i}}, \quad\left[u_{i}\right]_{S_{i-1}}=-V_{S_{i-1}},
$$

and the jumps of their derivatives

$$
\left[\partial_{\mathbf{n}} u_{i}\right]_{S_{i}}=\left(\partial_{\mathbf{n}} V\right)_{S_{i}}^{-}, \quad\left[\partial_{\mathbf{n}} u_{i}\right]_{S_{i-1}}=-\left(\partial_{\mathbf{n}} V\right)_{S_{i-1}}^{+} .
$$

We define $p_{S_{i}}=\sigma_{i}\left[\partial_{\mathbf{n}} u_{i}\right]_{S_{i}}=\sigma_{i}\left(\partial_{\mathbf{n}} V\right)_{S_{i}}^{-}$. For $i=1,2$, since $\left[\sigma \partial_{\mathbf{n}} V\right]=0$ from (4), we have $p_{S_{i}}=\sigma_{i}\left(\partial_{\mathbf{n}} V\right)_{S_{i}}^{-}=$ $\sigma_{i+1}\left(\partial_{\mathbf{n}} V\right)_{S_{i}}^{+}$at the interface $S_{i}$. The boundary condition (5) of EIT implies that $p_{S_{3}}$ is equal to the injected current $j$.

Let us first consider the inner surfaces $S_{1}$ and $S_{2}$. For $i=1,2$, the function $u_{i}$ is harmonic in $\mathbb{R}^{3} \backslash \partial \Omega_{i}$ and it satisfies the condition $\mathscr{H}$. We can therefore apply theorem 3.1 to obtain the internal limit of $u_{i}$ on $S_{i}$ :

$$
\left(u_{i}\right)_{S_{i}}^{-}=\frac{\left[u_{i}\right]_{\partial \Omega_{i}}}{2}-\mathcal{D}_{\partial \Omega_{i}}\left[u_{i}\right]_{\partial \Omega_{i}}+\delta_{\partial \Omega_{i}}\left[\partial_{\mathbf{n}} u_{i}\right]_{\partial \Omega_{i}}
$$




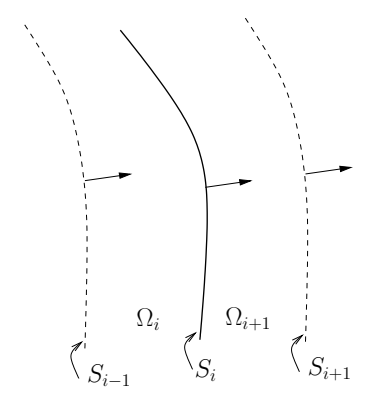

FIgure 2. A detail of the nested volume model. Normal vectors are oriented globally outward, as shown. However, when considering for example the surface $S_{i}$ as the boundary of $\Omega_{i+1}$, the orientation needs to be reversed.

If we break down the jump across $\partial \Omega_{i}=S_{i-1} \cup S_{i}$ into two parts, corresponding to $S_{i-1}$ and $S_{i}$, and if we take into account identities (7a), we obtain

$$
\left(u_{i}\right)_{S_{i}}^{-}=V_{S_{i}}^{-}=\frac{V_{S_{i}}}{2}+\mathcal{D}_{i, i-1} V_{S_{i-1}}-\mathcal{D}_{i i} V_{S_{i}}-\sigma_{i}^{-1} \mathcal{S}_{i, i-1} p_{S_{i-1}}+\sigma_{i}^{-1} \mathcal{S}_{i i} p_{S_{i}}
$$

Terms corresponding to $S_{0}=\emptyset$ must be set to 0 .

Theorem 3.1 gives the external limit of $u_{i+1}$ on $S_{i}$

$$
\left(u_{i+1}\right)_{S_{i}}^{+}=-\frac{\left[u_{i+1}\right]_{\partial \Omega_{i+1}}}{2}-\mathcal{D}_{\partial \Omega_{i+1}}\left[u_{i+1}\right]_{\partial \Omega_{i+1}}+\mathcal{S}_{\partial \Omega_{i+1}}\left[\partial_{\mathbf{n}} u_{i+1}\right]_{\partial \Omega_{i+1}}
$$

We substitute from $(7 \mathrm{a})$ for the values of $\left[u_{\Omega_{i+1}}\right]$ and $\left[\partial_{\mathbf{n}} u_{i+1}\right]$ and break down the terms on $\partial \Omega_{i+1}=S_{i} \cup S_{i+1}$, to obtain

$$
\left(u_{i+1}\right)_{S_{i}}^{+}=V_{S_{i}}^{+}=\frac{V_{S_{i}}}{2}+\mathcal{D}_{i i} V_{S_{i}}-\mathcal{D}_{i, i+1} V_{S_{i+1}}-\sigma_{i+1}^{-1} \mathcal{S}_{i i} p_{S_{i}}+\sigma_{i+1}^{-1} \mathcal{S}_{i, i+1} p_{S_{i+1}}
$$

Subtracting (8) and (9), and given that $V$ is continuous across $S_{i}$, we get for $i=1,2$,

$$
\mathcal{D}_{i, i-1} V_{S_{i-1}}-2 \mathcal{D}_{i i} V_{S_{i}}+\mathcal{D}_{i, i+1} V_{S_{i+1}}-\sigma_{i}^{-1} \mathcal{S}_{i, i-1} p_{S_{i-1}}+\left(\sigma_{i}^{-1}+\sigma_{i+1}^{-1}\right) \mathcal{S}_{i i} p_{S_{i}}-\sigma_{i+1}^{-1} \mathcal{S}_{i, i+1} p_{S_{i+1}}=0 .
$$

Using the same approach, we evaluate the quantities $\left(\sigma_{i} \partial_{\mathbf{n}} u_{i}\right)_{S_{i}}^{-}=p_{S_{i}}^{-}$and $\left(\sigma_{i+1} \partial_{\mathbf{n}} u_{i+1}\right)_{S_{i}}^{+}=p_{S_{i}}^{+}$using Theorem 3.1, subtract the resulting expressions and obtain for $i=1,2$,

$$
\sigma_{i} \mathcal{N}_{i, i-1} V_{S_{i-1}}-\left(\sigma_{i}+\sigma_{i+1}\right) \mathcal{N}_{i i} V_{S_{i}}+\sigma_{i+1} \mathcal{N}_{i, i+1} V_{S_{i+1}}-\mathcal{D}_{i, i-1}^{*} p_{S_{i-1}}+2 \mathcal{D}_{i i}^{*} p_{S_{i}}-\mathcal{D}_{i, i+1}^{*} p_{S_{i+1}}=0
$$

Here again, the terms corresponding to $S_{0}=\emptyset$ are to be set to zero. Terms involving $p_{S_{3}}$ are known quantities in the forward problem, and can be set to $j$.

Applying the Representation Theorem to $u_{3}$ we see that

$$
-p_{S_{3}}=\frac{-p_{S_{3}}}{2}+\mathcal{D}_{32}^{*} p_{S_{2}}-\mathcal{D}_{33}^{*} p_{S_{3}}-\sigma_{3} \mathcal{N}_{32} V_{S_{2}}+\sigma_{3} \mathcal{N}_{33} V_{S_{3}}
$$

Observe that the unknowns of a given surface only interact with unknowns of the neighboring surfaces. Equations (10)-(11)-(12) lead to a block-diagonal symmetric operator matrix 


$$
\left[\begin{array}{ccccc}
\left(\sigma_{1}+\sigma_{2}\right) \mathcal{N}_{11} & -2 \mathcal{D}_{11}^{*} & -\sigma_{2} \mathcal{N}_{12} & \mathcal{D}_{12}^{*} & 0 \\
-2 \mathcal{D}_{11} & \left(\sigma_{1}^{-1}+\sigma_{2}^{-1}\right) \mathcal{S}_{11} & \mathcal{D}_{12} & -\sigma_{2}^{-1} \mathcal{S}_{12} & 0 \\
-\sigma_{2} \mathcal{N}_{21} & \mathcal{D}_{21}^{*} & \left(\sigma_{2}+\sigma_{3}\right) \mathcal{N}_{22} & -2 \mathcal{D}_{22}^{*} & -\sigma_{3} \mathcal{N}_{23} \\
\mathcal{D}_{21} & -\sigma_{2}^{-1} \mathcal{S}_{21} & -2 \mathcal{D}_{22} & \left(\sigma_{2}^{-1}+\sigma_{3}^{-1}\right) \mathcal{S}_{22} & \mathcal{D}_{23} \\
0 & 0 & -\sigma_{3} \mathcal{N}_{32} & \mathcal{D}_{32}^{*} & \sigma_{3} \mathcal{N}_{33}
\end{array}\right] \cdot\left[\begin{array}{c}
V_{S_{1}} \\
p_{S_{1}} \\
V_{S_{2}} \\
p_{S_{2}} \\
V_{S_{3}}
\end{array}\right]=\mathrm{b}
$$

with

$$
\mathbf{b}=\left[\begin{array}{lllll}
0 & 0 & -\mathcal{D}_{23}^{*} j & \sigma_{3}^{-1} \mathcal{S}_{23} j & -\frac{j}{2}+\mathcal{D}_{33}^{*} j
\end{array}\right]^{T}
$$

The EEG case, for which $f \neq 0$ in (1) but $j=0$ in (2), has already been treated in [10]. It only differs from EIT by the right-hand side term b. As an example, supposing $f$ to be supported in $\Omega_{1}$, and $j$ in (2) to vanish on the scalp, then

$$
\mathbf{b}=\left[\begin{array}{lllll}
\left(\sigma_{1} \partial_{\mathbf{n}} v\right)_{S_{1}} & (v)_{S_{1}} & 0 & 0 & 0
\end{array}\right]^{T}
$$

where $v$ is the solution of $\sigma_{1} \Delta v=f$, in an infinite domain with conductivity $\sigma_{1}$, i.e. $v=-\frac{1}{\sigma_{1}} f * G$.

If both internal sources $f$ and injected current $j$ are present, the right-hand side terms (14) and (15) are simply summed.

\section{Discretization}

\subsection{Mixed finite elements}

The surfaces are represented by triangular meshes. Denoting $\psi_{i}^{(k)}$ the P0 function associated to triangle $i$ on surface $S_{k}$, and $\phi_{j}^{(l)}$ the P1 function associated to node $j$ on surface $S_{l}$, the potential $V$ on surface $S_{k}$ is approximated as $V_{S_{k}}(\mathbf{r})=\sum_{i} x_{i}^{(k)} \phi_{i}^{(k)}(\mathbf{r})$, while $p$ on surface $S_{k}$ is approximated by $p_{S_{k}}(\mathbf{r})=\sum_{i} y_{i}^{(k)} \psi_{i}^{(k)}(\mathbf{r})$.

The scalp current is discretized with $P 0$ elements on surface $S_{3}$ :

$$
j=\sum z_{i} \psi_{i}^{(3)}(\mathbf{r}) .
$$

The vectors $\left(\mathbf{x}_{k}\right)_{i}=x_{i}^{(k)},\left(\mathbf{y}_{k}\right)_{i}=y_{i}^{(k)}$, and $(\mathbf{z})_{i}=z_{i}$ are related by the discrete version of (13):

$$
\left[\begin{array}{ccccc}
\left(\sigma_{1}+\sigma_{2}\right) \mathrm{N}_{11} & -2 \mathrm{D}_{11}^{*} & -\sigma_{2} \mathrm{~N}_{12} & \mathrm{D}_{12}^{*} & 0 \\
-2 \mathrm{D}_{11} & \left(\sigma_{1}^{-1}+\sigma_{2}^{-1}\right) \mathrm{S}_{11} & \mathrm{D}_{12} & -\sigma_{2}^{-1} \mathrm{~S}_{12} & 0 \\
-\sigma_{2} \mathrm{~N}_{21} & \mathrm{D}_{21}^{*} & \left(\sigma_{2}+\sigma_{3}\right) \mathrm{N}_{22} & -2 \mathrm{D}_{22}^{*} & -\sigma_{3} \mathrm{~N}_{23} \\
\mathrm{D}_{21} & -\sigma_{2}^{-1} \mathrm{~S}_{21} & -2 \mathrm{D}_{22} & \left(\sigma_{2}^{-1}+\sigma_{3}^{-1}\right) \mathrm{S}_{22} & \mathrm{D}_{23} \\
0 & 0 & -\sigma_{3} \mathrm{~N}_{32} & \mathrm{D}_{32}^{*} & \sigma_{3} \mathrm{~N}_{33}
\end{array}\right]\left[\begin{array}{c}
\mathbf{x}_{1} \\
\mathbf{y}_{1} \\
\mathbf{x}_{2} \\
\mathbf{y}_{2} \\
\mathbf{x}_{3}
\end{array}\right]=\left[\begin{array}{c}
0 \\
0 \\
-\mathrm{D}_{23}^{*} \mathbf{z} \\
\sigma_{3}^{-1} \mathbf{D}_{23} \mathbf{z} \\
\left(-\frac{1}{2} l_{33}+\mathrm{D}_{33}^{*}\right) \mathbf{z}
\end{array}\right]
$$

Each block is a submatrix, representing the interactions between test functions on a given pair of surfaces $S_{k}$ and $S_{l}$. More precisely,

$$
\begin{array}{ll}
\left(\mathrm{N}_{k l}\right)_{i j}=\left\langle\mathcal{N}_{k l} \phi_{j}^{(l)}, \phi_{i}^{(k)}\right\rangle & \left(\mathrm{S}_{k l}\right)_{i j}=\left\langle\mathcal{S}_{k l} \psi_{j}^{(l)}, \psi_{i}^{(k)}\right\rangle \\
\left(\mathrm{D}_{k l}\right)_{i j}=\left(\mathrm{D}_{l k}^{*}\right)_{j i}=\left\langle\mathcal{D}_{k l} \phi_{j}^{(l)}, \psi_{i}^{(k)}\right\rangle & \left(\mathrm{I}_{k k}\right)_{i j}=\left\langle\psi_{i}^{(k)}, \phi_{j}^{(k)}\right\rangle
\end{array}
$$

The potential $V$ being only defined up to a constant, the above system has an indeterminacy, which is lifted by deflating the last block $\sigma_{3} \mathrm{~N}_{33}[3,4,15]$. 


\subsection{Validation}

To validate the EIT forward problem, we use the principle of reciprocity [13], a direct consequence of the Green identity $\int_{\Omega} \nabla \cdot \vec{a}=\int_{\partial \Omega} \vec{a} \cdot \mathbf{n}$.

We introduce two potentials: $V_{f}$ the solution of an EEG forward problem (1)-(2) with $f=\nabla \cdot \vec{J}_{p}$ and $j=0$, and $V_{j}$ the solution of an EIT forward problem (1)-(2) with $f=0$ and $j=\delta_{r_{i}}-\delta_{r_{e}}$. Applying the Green identity to $\vec{a}=\sigma V_{j} \nabla V_{f}$, we obtain

$$
\int_{\Omega}\left(\nabla \cdot \sigma \nabla V_{f}\right) V_{j}+\int_{\Omega} \sigma \nabla V_{f} \cdot \nabla V_{j}=\int_{\partial \Omega} \sigma V_{j} \partial_{\mathbf{n}} V_{f}
$$

and the same relationship holds when exchanging the roles of $V_{f}$ and $V_{j}$. Combining the difference of the two relationships with equations (1)-(2):

$$
\int_{\Omega} f V_{j}=\int_{\partial \Omega} j V_{f}
$$

Given the forms of the injection current $j=\delta_{r_{i}}-\delta_{r_{e}}$ and of the EEG source term $f=\nabla \cdot \vec{J}_{p}$,

$$
\int_{\Omega} \vec{J}_{p}(r) \cdot \nabla V_{j}=V_{f}\left(r_{i}\right)-V_{f}\left(r_{e}\right)
$$

This shows that the gradient of the EIT potential, $\nabla V_{j}$, can be viewed as a lead field for EEG. We have used this observation to validate the EIT solution $V_{j}$, for the case of a dipolar EEG source $\vec{J}_{p}(r)=\vec{q} \delta_{r_{0}}$; first on concentric spheres, with an analytical EEG solution, and then also for a realistic head geometry model, with the forward EEG solution $V_{f}$ calculated with the symmetric BEM.

\section{Results}

A series of $M$ experiments was performed on the same human subject. In each experiment $m=1, \ldots, M$, we noted

- the electrode labels $I_{m}$ used for current injection,

- the potential $v_{m}$ measured on the remaining electrodes.

The potential could not be reliably measured on the injection and extraction electrodes because of the impedances involved. A magnetic resonance (MR) image of the subject's head was segmented into three surfaces using ASA (Advanced Source Analysis, ANT). The meshes generated were of sufficient quality for BEM and did not require any post-processing. The number of vertices per surface was 510 (brain-skull), 510 (skull-scalp) and 1222 (scalp-air). We assume the intensity of the current injection to be known. For a fixed conductivity distribution $\boldsymbol{\sigma}=\left(\sigma_{1}, \sigma_{2}, \sigma_{3}\right)$, the forward EIT problem was solved using the symmetric BEM proposed in this article, yielding a potential $V\left(\boldsymbol{\sigma}, j_{m}\right)$ on the scalp. Figure 3 shows the scalp potentials simulated for two current injection patterns $j_{1}$ and $j_{2}$ on the scalp corresponding to two of the patterns applied in the experiments.

The goal of the inverse EIT problem is to find a conductivity distribution $\boldsymbol{\sigma}=\left(\sigma_{1}, \sigma_{2}, \sigma_{3}\right)$ minimizing a cost function, which measures the discrepancy between simulations and measurements:

$$
E(\boldsymbol{\sigma})=\frac{1}{2} \sum_{m=1}^{M}\left\|V\left(\boldsymbol{\sigma}, j_{m}\right)-v_{m}\right\|_{\text {meas }}^{2} .
$$

The norm \|\|$_{\text {meas }}^{2}$ is a discrete $\ell^{2}$ norm over the measurement electrodes: denoting $r_{k}$ the position of electrode $k$,

$$
\left\|V-v_{m}\right\|_{\text {meas }}^{2}=\sum_{k \notin I_{m}}\left|V\left(r_{k}\right)-v_{m}\left(r_{k}\right)\right|^{2} .
$$



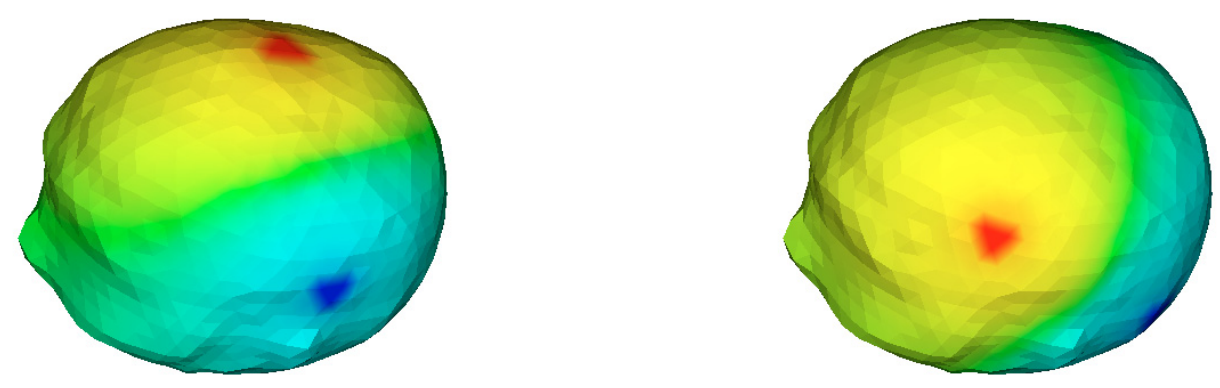

Figure 3. Simulation results for the EIT forward problem. The potential on the scalp is depicted, for two different current injection patterns $j_{1}$ and $j_{2}$, corresponding to two different pairs of electrodes.

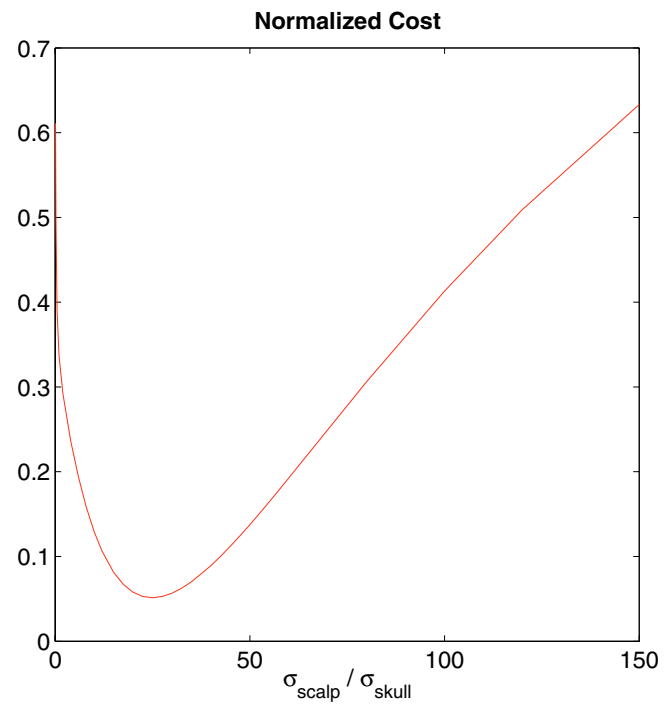

Figure 4. The cost function $E(\boldsymbol{\sigma})$ measures the discrepancy between simulated and measured potentials on the scalp. It displays a clear minimum when plotted as a function of the scalpto-skull conductivity ratio.

Figure 3 displays the cost function (19), normalized by $\frac{1}{2} \sum_{m=1}^{M}\left\|v_{m}\right\|_{\text {meas }}^{2}$, when the skull conductivity varies and the brain and scalp conductivities remain constant.

The cost function displays a clear minimum when the ratio of the scalp conductivity to the skull conductivity is approximately $\sigma_{3} / \sigma_{2}=25$. This value is in agreement with recent results in the literature $[1,5-7,12]$. The 
EIT setup thus appears well-suited for skull conductivity estimation. From our numerical experiments, varying the brain conductivity only modifies the cost function very little, and EIT with injected current on the scalp hence seems less appropriate for estimating brain conductivity than skull conductivity.

We are working on the resolution of the inverse EIT problem, and we plan to perform more experiments on different subjects to further validate the method.

The authors would like to thank Patrick Marquis for designing the data acquisition procedure. This work is partially supported by the French Research Ministry ACI Masse de Données Obs-Cerv, and the Czech Ministry of Education under project MSM6840770012. The CEMRACS research center at Luminy was a wonderful opportunity for scientific exchanges and the authors are particularily grateful to Eric Cancès and Jean-Frédéric Gerbeau for their personal involvement in organizing this event.

\section{REFERENCES}

[1] U. Baysal and J. Haueisen. Use of a priori information in estimating tissue resistivities - application to human data in vivo. Physiol. Meas., 25:737-748, 2004.

[2] Marc Bonnet. Equations intégrales et éléments de frontière. CNRS Editions, Eyrolles, 1995.

[3] T. F. Chan. Deflated decomposition solution of nearly singular systems. SIAM J. Numer. Anal., 21:739-754, 1984.

[4] G. Fischer, B. Tilg, R. Modre, F. Hanser, B. Messnarz, and P. Wach. On modeling the Wilson terminal in the Boundary and Finite Element Method. IEEE Trans. Biomed. Eng., 49(3):217-224, March 2002.

[5] S. Gonçalves, J.C. de Munck, J.P. Verbunt, R.M. Heethaar, and F.H. Lopes da Silva. In vivo measurement of the brain and skull resistivities using an EIT-based method and the combined analysis of SEF/SEP data. IEEE Transactions on Biomedical Engineering, 50(9):1124-8, September 2003.

[6] Sonia I. Gonçalves, Jan C. de Munck, Jeroen P.A. Verbunt, Fetsje Bijma, Rob M. Heethaar, and Fernando Lopes da Silva. In vivo measurement of the brain and skull resistivities using an EIT-based method and realistic models for the head. IEEE Transactions on Biomedical Engineering, 50(6), 2003.

[7] David Guttiérrez, Arye Nehorai, and Carlos H. Muravchik. Estimating brain conductivities and dipole source signals with EEG arrays. IEEE Transactions on Biomedical Engineering, 51(12):2113-2122, December 2004.

[8] Matti Hämäläinen, Riitta Hari, Risto J. IImoniemi, Jukka Knuutila, and Olli V. Lounasmaa. Magnetoencephalographytheory, instrumentation, and applications to noninvasive studies of the working human brain. Reviews of Modern Physics, 65(2):413-497, April 1993.

[9] G. Huiskamp, M. Vroeijenstijn, R. van Dijk, G. Wieneke, and A. C. van Huffelen. The need for correct realistic geometry in the inverse EEG problem. IEEE Trans. on Biomed. Engin., 46(11):1281-1287, November 1999.

[10] J. Kybic, M. Clerc, T. Abboud, O. Faugeras, R. Keriven, and T. Papadopoulo. A common formalism for the integral formulations of the forward EEG problem. IEEE Transactions on Medical Imaging, 24(1):12-28, January 2005.

[11] Jean-Claude Nédélec. Acoustic and Electromagnetic Equations. Springer Verlag, 2001.

[12] T.F. Oostendorp, J. Delbeke, and D.F. Stegeman. The conductivity of the human skull: Results of in vivo and in vitro measurements. IEEE Transactions on Biomedical Engineering, 47(11):1487-1492, December 2000.

[13] R. Plonsey. Reciprocity applied to volume conductors and the ECG. IEEE Trans. on Biomed. Engin., 10(1):9-12, January 1963.

[14] Jukka Sarvas. Basic mathematical and electromagnetic concepts of the biomagnetic inverse problem. Phys. Med. Biol., 32(1):1122, 1987.

[15] Satu Tissari and Jussi Rahola. Error analysis of a new Galerkin method to solve the forward problem in MEG and EEG using the boundary element method. Technical Report TR/PA/98/39, CERFACS, 1998. Toulouse, France. 